Revue internationale P.M.E.

Économie et gestion de la petite et moyenne entreprise

Revue

internationale

PME

\title{
L'entrepreneuriat comme processus
}

\section{Émile-Michel Hernandez}

Volume 8, numéro 1, 1995

URI : https://id.erudit.org/iderudit/1008277ar

DOI : https://doi.org/10.7202/1008277ar

Aller au sommaire du numéro

Éditeur(s)

Presses de l'Université du Québec

ISSN

0776-5436 (imprimé)

1918-9699 (numérique)

Découvrir la revue

Citer cette note

Hernandez, É.-M. (1995). L'entrepreneuriat comme processus. Revue internationale P.M.E., 8(1), 107-119. https://doi.org/10.7202/1008277ar

\section{Résumé de l'article}

La recherche en sciences de gestion a connu trois grandes périodes : le fondamentalisme (recherche de la bonne et unique solution), la contingence (l'efficacité dépend de l'adaptation et de la cohérence du concept et de la situation), et le processus (les concepts ne correspondent plus à des notions discrètes mais à des phénomènes dont l'évolution même constitue le sens). Cet article se propose de présenter les recherches récentes en entrepreneuriat relevant d'une approche axée sur le processus, et de montrer ce qu'elles apportent pour une meilleure connaissance et compréhension du phénomène entrepreneurial.
Ce document est protégé par la loi sur le droit d'auteur. L'utilisation des services d'Érudit (y compris la reproduction) est assujettie à sa politique d'utilisation que vous pouvez consulter en ligne.

https://apropos.erudit.org/fr/usagers/politique-dutilisation/ 


\title{
L'entrepreneuriat comme processus
}

\author{
Émile-Michel HERNANDEZ*
}

IUT de Saint-Nazaire

\section{MOTS CLÉS}

\section{Comportement entrepreneurial - Création d'entreprise \\ Créateur d'entreprise - Entrepreneuriat - Intrapreneuriat \\ Organisation émergente - Processus entrepreneurial}

\begin{abstract}
RÉSUMÉ
La recherche en sciences de gestion a connu trois grandes périodes: le fondamentalisme (recherche de la bonne et unique solution), la contingence (l'efficacité dépend de l'adaptation et de la cohérence du concept et de la situation), et le processus (les concepts ne correspondent plus à des notions discrètes mais à des phénomènes dont l'évolution même constitue le sens). Cet article se propose de présenter les recherches récentes en entrepreneuriat relevant d'une approche axée sur le processus, et de montrer ce qu'elles apportent pour une meilleure connaissance et compréhension du phénomène entrepreneurial.
\end{abstract}

\begin{abstract}
Research in management sciences has known three great periods: fundamentalism (research of the good and unique solution), contingency (efficiency depends on the adaptation and coherence of the concept and of the situation) and process (concepts no longer correspond to discrete notions but to phenomena whose very evolution constitutes their meaning). This article aims at presenting the recent research in entrepreneurship with an approach in terms of process and at showing what they bring for a better knowledge and understanding of the entrepreneurial phenomenon.
\end{abstract}

\section{RESUMEN}

La investigación en ciencias de gestión conoció tres grandes períodos : el fondamentalismo (búsqueda de la buena y única solución), la contingencia (la eficacia depende de la adaptación y la coherencia del concepto y de la

* Émile-Michel Hernandez est docteur en économie et administration des entreprises, docteur d'État en sciences de gestion, maître de conférences en sciences de gestion à l'Université de Nantes. Travaux de recherche consacrés à l'analyse du phénomène entrepreneurial et à ses caractéristiques dans les pays en voie de développement. Adresse : IUT de Saint-Nazaire, Département techniques de commercialisation, B.P. 420, Heinlex, 44606 Saint-Nazaire (France). 
situación) y el procedimiento (los conceptos ya no corresponden a nociones discretas sino a fenómenos cuya evolución constituye en si el sentido). El propósito de este artículo consiste en presentar las recientes investigaciones empresariales relativas a su acercamiento en lo que toca al procedimiento y de mostrar que contribuyen a un conocimiento más amplio y a una comprensión del fenómeno empresarial.

\section{Introduction}

La création d'entreprises reste en France un thème très mineur pour les sciences de gestion. Peu de chercheurs s'y intéressent et l'enseignement, à la notable exception de quelques écoles de commerce, en est quasi inexistant. Aux États-Unis, la situation est totalement différente. La plupart des universités possèdent des chaires d'entrepreneuriat et de nombreux cours sont offerts aux étudiants intéressés. Parallèlement à cet effort d'enseignement, la recherche s'est développée et de nombreux travaux ont été publiés. Pourtant, il faut bien constater aujourd'hui que les résultats obtenus ne sont pas à la hauteur des efforts fournis et des espoirs entretenus par nos collègues américains. Aussi trouve-t-on dans les publications récentes une remise en cause des recherches passées et une volonté de réorientation des travaux actuels.

Pourquoi une telle situation ? Les premiers chercheurs ont essayé de répondre à deux questions. Tout d'abord, ils ont tenté d'identifier les créateurs par opposition à ceux qui ne créent pas, de repérer dans une population ceux qui étaient le plus susceptibles de créer une entreprise. Puis ils ont cherché des critères pour séparer les créateurs qui réussissent de ceux qui échouent. On comprend l'intérêt pour une économie, surtout en période de crise, de disposer d'un moyen infaillible pour détecter les futurs créateurs d'entreprises performantes et les inciter à se mettre au travail. Il faut bien avouer que, pour le moment, la recherche de cette pierre philosophale a échoué.

Diverses raisons peuvent expliquer cette remise en cause : la focalisation des recherches effectuées, les méthodologies utilisées, etc. Une autre raison peut également être invoquée : les chercheurs en entrepreneuriat ont suivi le même cheminement que l'ensemble des chercheurs en sciences de gestion. On peut le décomposer en trois grandes étapes. La première, qualifiée par Thévenet et Vachette (1992) de « fondamentalisme », consiste en une conception très unitaire du concept abordée selon une logique unique. Il s'agit de distinguer les caractéristiques permanentes du succès, la bonne façon de procéder. En entrepreneuriat, la recherche du profil du créateur qui réussit est caractéristique de cette démarche.

La deuxième étape est celle de la contingence. Elle relie l'efficacité à l'adaptation, ainsi qu'à la cohérence du concept et de la situation. Il n'existe 
plus de solution universelle, mais seulement des solutions appropriées aux caractéristiques du monde des entreprises. Selon Mérigot (1992) : « réfutant l'universalisme mais évitant tout autant les excès d'une perspective situationnelle dans laquelle chaque situation est unique, elle [la théorie de la contingence] retient la possibilité de dégager des analogies parmi certaines catégories de situations qu'elle regroupe, et souligne que la valeur d'un principe de management est "contingente" à une configuration de facteurs et à leurs interrelations dans le cadre de ces situations types ainsi définies ». En entrepreneuriat, les chercheurs ont mesuré l'extrême variété des situations de création et des entités créées. Ils se sont aperçus qu'il y avait parfois plus de différences entre deux créateurs qu'entre un créateur et un non-créateur.

La troisième étape est l'approche axée sur le processus. Elle rompt totalement avec les deux précédentes. Ici, les concepts ne correspondent plus à des notions discrètes, isolables dans le temps, mais recouvrent des phénomènes en évolution et dont l'évolution même constitue le sens. Pour Thévenet et Vachette, cette approche du processus met l'accent sur deux aspects principaux. La relation et l'interaction comptent plus que les éléments eux-mêmes. Cette relation est évolutive dans le temps, le processus renvoie à une approche diachronique de l'organisation et de son fonctionnement qui s'oppose aux approches fixistes du monde où l'on fige des relations et l'état des notions et des concepts. En entrepreneuriat, elle correspond à toutes ces publications récentes où il n'est plus question du créateur et de ses caractéristiques, mais de formation d'organisation, de création d'organisation, d'émergence organisationnelle...

Deux auteurs, Bygrave et Hofer (1991), ont traduit ce changement de centre d'intérêt en entrepreneuriat, ce passage du fondamentalisme au processus.

\section{Quelques questions clés en entrepreneuriat}

$\begin{array}{ll}\text { Centrées sur l'entrepreneur } & \begin{array}{l}\text { Centrées sur le processus } \\ \text { entrepreneurial }\end{array}\end{array}$

1. Qui devient entrepreneur?

2. Pourquoi devient-on entrepreneur?

3. Quelles sont les caractéristiques des entrepreneurs qui réussissent?

4. Quelles sont les caractéristiques des entrepreneurs qui échouent?
1. Qu'est-ce qui permet de percevoir les occasions d'une manière efficace et performante ?

2. Quelles sont les tâches clés pour créer avec succès de nouvelles organisations?

3. Dans quelle mesure ces tâches sontelles différentes de celles mises en œuvre pour diriger avec succès ?

4. Quelles sont les contributions directes de l'entrepreneur dans ce processus? 
Un article de Gartner (1989) marque un tournant dans la recherche. Ayant constaté l'insuccès des travaux portant sur les caractéristiques du créateur, il propose de cesser de s'intéresser à la personne de l'entrepreneur pour regarder ce qu'il fait, son comportement : "La recherche sur l'entrepreneur devrait se focaliser sur ce que fait l'entrepreneur et non sur ce qu'il est. » Dans cette approche comportementale, la création d'une organisation est un événement contextuel, le résultat de nombreuses influences et l'entrepreneur intervient dans ce processus complexe. Ses actions ont pour résultat la création d'une organisation. Pour Gartner, la meilleure façon d'étudier le comportement entrepreneurial est de procéder comme Mintzberg l'a fait avec le comportement managérial : "Je crois que la recherche sur les comportements entrepreneuriaux doit être fondée sur le travail de terrain selon le modèle de l'étude de Mintzberg sur le travail managérial. Les chercheurs doivent observer les entrepreneurs lorsqu'ils sont en train de créer des organisations. Ce travail doit être décrit en détail et les activités systématisées et classifiées. La connaissance des comportements entrepreneuriaux dépend du travail de terrain. »

À la fin des années 80 , et surtout au début des années 90 , sont publiés un certain nombre de travaux de recherches où la création d'entreprises cesse d'être analysée comme la photographie instantanée d'un événement où le créateur est d'abord seul (approche par les caractéristiques du créateur), puis n'est plus seul, mais joue toujours le rôle principal (modèles de Shapero et al., puis contingence). Elle devient un film dont le créateur est un des acteurs; c'est l'approche axée sur le processus entrepreneurial.

Bull et Willard (1993) rappellent l'extrême hétérogénéité des recherches portant sur l'entrepreneuriat et les origines diverses des chercheurs : « Des universitaires, spécialistes de domaines aussi divers que l'agriculture, l'anthropologie, l'économie, l'éducation, la finance, l'histoire, le marketing, la communication, la science politique, la psychologie, la sociologie et la stratégie, ont fait paraître des articles et sont ainsi venus grossir le corps de chercheurs déjà existant. »

L'approche centrée sur le processus de l'entrepreneuriat fait, pour sa part, essentiellement référence à des notions issues de la théorie des organisations : d'une part, le comportement organisationnel et, d'autre part, la notion d'émergence organisationnelle.

\section{Le comportement entrepreneurial}

Un certain nombre de travaux, datant en général du début des années 90 , utilisent, pour analyser le phénomène d'entrepreneuriat, des notions issues du champ appelé «comportement organisationnel». Ce terrain de réflexion 
concerne principalement les rapports entre les individus et les organisations, ainsi que les rapports interindividuels ou intergroupes au sein des organisations. Il est structuré autour de quatre grands thèmes : l'engagement de l'individu dans l'action et l'organisation (notions de motivation, d'implication), l'adéquation individu-organisation (modes d'ajustement portant sur l'échange d'informations, la confrontation des valeurs, l'apprentissage dans l'organisation), les dynamiques de «leadership », et les relations interpersonnelles et intergroupes.

Plusieurs aspects de l'entrepreneuriat sont ainsi étudiés en se référant au champ du comportement organisationnel : la décision proprement dite de créer une entreprise, le comportement dans une organisation en cours de création, le comportement entrepreneurial dans une organisation existante appelé « intrapreneuriat».

\subsection{La décision de créer}

Greenberger et Sexton (1988) proposent un modèle interactif de création d'une nouvelle organisation. Fondé sur les résultats de recherches de psychologie du comportement des entrepreneurs, ce modèle émet l'hypothèse selon laquelle la décision de création d'une nouvelle entreprise est basée sur l'interaction d'un certain nombre de facteurs parmi lesquels peuvent être inclus l'esprit d'entreprise, le désir de liberté de décision, certains traits de personnalité, des variables de situation, la connaissance de soi et le soutien social.

Ce modèle a trois composantes principales. D'abord, trois facteurs sont censés servir de catalyseur dans l'identification de l'opportunité de créer une nouvelle entreprise : la vision entrepreneuriale (c'est-à-dire l'image de ce que le créateur veut réaliser), sa personnalité, le contrôle souhaité (le contrôle personnel étant la perception qu'a l'individu d'une relation entre ses actions et les résultats souhaités). Ces trois facteurs agissent seuls ou ensemble pour augmenter la probabilité qu'a un individu de créer une nouvelle entreprise. Puis quatre différents facteurs influent sur l'effet de ces catalyseurs sur les décisions individuelles; ils vont aider à faire passer un individu de l'état de non-créateur à celui de créateur : le fait d'avoir vécu des événements significatifs sur le plan entrepreneurial, se percevoir soi-même comme le genre de personne qui crée une entreprise, disposer d'un soutien social (famille, amis, relations...), voir la création comme un moyen satisfaisant d'obtenir et d'augmenter le contrôle désiré. Enfin, la troisième composante, c'est la probabilité qu'a un individu de créer une nouvelle entreprise.

Pour leur part, Guth, Kumaraswamy et McErlean (1991) ont mené une étude longitudinale sur le processus cognitif d'un entrepreneur en train de créer une nouvelle unité. Ils se sont en particulier intéressés à la façon dont le 
créateur analyse ses chances de succès, au décalage entre ce qu'il estime être ses propres chances ( $80 \%$ des entrepreneurs les estiment à $70 \%$ ou plus et un tiers les considèrent comme certaines), les chances des autres dans une activité comparable à la leur $(59 \%)$ et la réalité (de $10 \%$ à $40 \%$ seulement des nouvelles entreprises ont une chance de survie au bout de cinq ans d'existence). Il s'agit de chiffres fournis par ces trois auteurs. Ils sont assez pessimistes, puisque, pour la France, le taux de survie à cinq ans est, selon l'INSEE, de $50 \%$ environ. Leur modèle de processus entrepreneurial considère que le succès est influencé de façon déterminante par le degré de congruence entre le schéma cognitif de l'entrepreneur, c'est-à-dire l'environnement tel qu'il le perçoit, et la réalité, ainsi que par son aptitude à tirer profit de son expérience, pour réajuster ce schéma cognitif à la réalité.

\subsection{Le travail des dirigeants et celui des créateurs}

Gartner, Bird et Starr (1992), eux, se sont intéressés aux différences de comportements des acteurs dans les organisations naissantes et dans les organisations existantes. Et à ce propos, ils rappellent les nombreuses études réalisées sur le travail des dirigeants et suggèrent aux chercheurs en entrepreneuriat de s'en inspirer en particulier sur le plan méthodologique. Ces études comportent certes des faiblesses : l'absence de progrès réalisés dans la définition de ce qui est managérial, dans la séparation entre le travail managérial et celui qui ne l'est pas et, enfin, dans l'étude des relations entre la nature du travail managérial et les performances de l'organisation.

Malgré ces quelques faiblesses, ils recommandent aux chercheurs en entrepreneuriat de s'inspirer de leurs collègues ayant étudié le travail des dirigeants : «En résumé, si l'étude du travail des dirigeants peut servir de modèle quant à la manière dont le domaine de l'entrepreneuriat pourrait générer une meilleure théorie, c'est en offrant une ouverture vers l'utilisation de plus de méthodologies pour décrire les activités spécifiques des individus [...] Les théories sur un phénomène exigent des informations sur les éléments qui composent ce phénomène proprement dit. » Les théories sur le comportement entrepreneurial ne pourront que tirer profit de la réalisation de nombreuses études utilisant diverses méthodes de collecte de données et décrivant ce que les entrepreneurs font.

\subsection{L'intrapreneuriat}

Covin et Slevin (1991) ont étudié le comportement entrepreneurial d'une organisation existante et ils proposent un modèle conceptuel d'entrepreneuriat comme phénomène d'origine organisationnelle. Pour ces auteurs, le 
comportement de la firme est l'élément central et essentiel du processus entrepreneurial. Le modèle proposé par ces deux auteurs fait ressortir les origines et les conséquences d'une attitude entrepreneuriale, ainsi que les variables qui influent sur les relations entre l'attitude entrepreneuriale et les performances de la firme.

Ce modèle présentant l'entrepreneuriat comme un comportement organisationnel a plusieurs conséquences.

1. On peut parler de comportement entrepreneurial pour une organisation comme pour un individu. Limiter l'étude de l'entrepreneuriat et du processus entrepreneurial aux seuls individus est donc une limitation indue.

2. Le comportement entrepreneurial peut être partie intégrante des activités de l'organisation. L'attitude entrepreneuriale devient ainsi une dimension stratégique de la firme.

3. Si l'attitude entrepreneuriale est un phénomène comportemental, cela veut dire qu'elle peut être gérée, favorisée ou contrariée (une attitude entrepreneuriale n'étant pas toujours souhaitable pour une entreprise).

4. L'attitude entrepreneuriale affecte les composantes de l'organisation et est affectée par elles. Il faut donc tenir compte de la structure organisationnelle, de la culture de l'entreprise, de ses ressources, de ses compétences... qui, indirectement, peuvent encourager ou au contraire gêner le comportement entrepreneurial de la firme.

Ce thème de l'intrapreneuriat a également été étudié par Hornsby, Naffziger, Kuratko et Montagno (1993). Ils en proposent un modèle qualifié « d'interactif » car, pour ces auteurs, l'intrapreneuriat est issu de la conjonction de trois grandes catégories de facteurs : des caractéristiques organisationnelles, des caractéristiques individuelles et un événement jouant un rôle de catalyseur. Se référant à Gartner (1989), pour qui la compréhension du processus entrepreneurial est plus importante que celle de l'entrepreneur, ils considèrent que la compréhension de l'intrapreneur constitue juste une partie de celle du processus intrapreneurial. Sa nature interactive ne doit pas être sousestimée, l'intrapreneuriat étant multidimensionnel et reposant plus sur l'interaction réussie de plusieurs facteurs que sur des événements isolés.

\section{La notion d'organisation émergente}

L'accent mis sur le processus entrepreneurial a amené les chercheurs à s'intéresser à la notion d'organisation émergente. C'est l'occasion d'un rapprochement avec la théorie des organisations, ces deux champs des sciences de 
gestion étant dans l'ensemble restés assez éloignés l'un de l'autre. La théorie des organisations s'est plus intéressée aux entités importantes et à l'existence bien affirmée qu'aux petites unités ou à celles en cours de création. Elle commence, en général, où l'organisation cesse d'être émergente. Trois thèmes seront successivement abordés : la présentation de cette nouvelle approche pour étudier l'entrepreneuriat et ses conséquences, en particulier concernant le vocabulaire utilisé, l'étude de la formation de l'organisation et, enfin, les caractéristiques des organisations émergentes.

\subsection{La nouvelle approche}

Gartner (1993) rappelle l'influence du vocabulaire utilisé sur la pensée, et même sur l'action. Pour cet auteur, le renouvellement de l'étude de l'entrepreneuriat doit aller de pair avec celui du vocabulaire utilisé pour le décrire. Il propose un petit glossaire de l'émergence organisationnelle avec, pour chaque terme, la définition correspondante : « être, situation, émerger, émergence, circonstance, évolution émergente, équivoque, trouvé, sombrer, genèse, variation ».

Dans cette optique étudier l'entrepreneuriat revient à étudier la naissance de nouvelles organisations, autrement dit les activités permettant à quelqu'un de créer une nouvelle entité plutôt que celles liées au développement, à la maintenance ou au changement d'unités existantes. Si on interroge les gens pour savoir ce qui s'est passé, on aura des réponses différentes selon qu'on s'adresse aux acteurs eux-mêmes ou à de simples observateurs. Les premiers attribuent les résultats positifs obtenus essentiellement à des causes internes (leurs propres actions et pensées) ; quant aux échecs, ils sont par contre dus à des causes externes (l'environnement). Les observateurs, eux, ont tendance à sous-estimer l'influence des facteurs externes ou personnels lorsqu'ils jugent le comportement d'autres individus.

Les créateurs de l'organisation jouent certes un rôle important dans son émergence mais s'intéresser moins à eux (contrairement à ce qui a été trop souvent fait dans les recherches) permet d'étudier d'autres facteurs intéressants et ayant aussi un rôle critique à jouer dans le phénomène.

L'émergence organisationnelle a lieu avant que l'organisation n'existe. Ce n'est pas une nouvelle entité, mais le processus dont le résultat est une nouvelle entité : «Si l'émergence survient avant que l'organisation n'existe, alors le phénomène n'est pas une nouvelle organisation. L'émergence organisationnelle est le processus d'organisation qui mène à une nouvelle organisation. » 
L'analyse de l'entrepreneuriat sous cet angle amène le chercheur à essayer de répondre aux questions suivantes : Comment naissent les organisations? Pourquoi ? Où ? Quand (et quand certaines activités se produisent-elles dans le processus d'émergence) ? Qui était impliqué et pourquoi, quand ces organisations sont nées?

Enfin le délicat problème de savoir qui est un entrepreneur et qui n'en est pas un devient pour Gartner (1993) facile à résoudre : « Ma réponse à ceux qui me demandent : Suis-je un entrepreneur ? est : Si vous lancez une organisation, vous êtes un entrepreneur, si vous n'en lancez pas une, vous n'en êtes pas un. Nous sommes ce que nous faisons. »

\subsection{La formation de l'organisation}

Tous les individus n'ont pas le potentiel pour créer une organisation. Ceux qui l'ont n'essaient pas tous ; et parmi ceux qui essaient, tous ne réussissent pas. Learned (1992) propose un modèle processuel de formation d'organisation qui tente de traduire ces observations. Ce phénomène pouvant revêtir de nombreux aspects, le modèle a pour objectif de permettre cette diversité plutôt que de se limiter à un aspect particulier. Il comporte trois dimensions du processus de création qui aboutissent in fine à la décision de créer ou non une organisation.

1. La propension à créer : certains individus ont une combinaison de caractéristiques psychologiques et un passé professionnel qui les rendent plus susceptibles d'essayer de créer une affaire que d'autres.

2. L'intention de créer : parmi ceux-ci certains rencontrent des situations qui déclenchent l'intention de créer.

3. La structuration des informations : cette étape consiste à rechercher et à trouver dans l'environnement les informations significatives, et à en déduire les tâches à exécuter pour créer l'organisation.

La décision : l'individu qui a l'intention de créer va, en fin de compte, en fonction de ces informations, se décider à agir ou non. Ce modèle met en évidence ce qui se passe avant que l'organisation n'existe, il correspond tout à fait à la conception qu'a Gartner de la notion d'émergence organisationnelle et qui a été développée précédemment.

\subsection{Les caractéristiques des organisations émergentes}

Katz et Gartner (1988) se demandent ce qu'est une organisation et quelles propriétés elle doit posséder pour exister. Pour ces auteurs, étudier l'organisation émergente, c'est s'intéresser à ce qui est compris entre la 
préorganisation et la nouvelle organisation. Pour définir l'organisation, ils se réfèrent à la définition donnée par McKelvey dans son ouvrage Organizational Systematics : «Une organisation est un système d'activité finalisé (se maintenant dans des limites) contenant un ou plusieurs sous-systèmes conditionnellement autonomes et finalisés ayant des ratios de ressources entrées-sorties permettant de survivre dans des environnements imposant des contraintes particulières. » Elle fait ressortir quatre propriétés principales des organisations : deux processuelles, l'intention et l'échange, et deux structurelles, les ressources et les limites. Ces quatre propriétés caractérisent une organisation complète, mais il est bien évident qu'elles n'apparaissent pas toutes simultanément ; souvent l'intention précède les trois autres. Pendant cette période où ces quatre propriétés apparaissent successivement puis se mettent à interagir, on peut dire qu'il y a plus que du hasard, mais moins qu'une organisation.

L'intention ou la volonté est le premier élément nécessaire pour créer une organisation. Elle reflète l'objectif ou les objectifs du ou des créateurs. En général, elle se traduit par la recherche de l'information utile pour agir.

Les ressources correspondent aux éléments qui, combinés, vont donner l'organisation. L'école de la dépendance sur les ressources a mis en évidence l'importance de cette notion. En général, elles consistent en moyens financiers (le capital et les crédits), humains (le personnel), matériel (bâtiments, machines, matières premières).

La notion de limite, plus abstraite, est également très importante. Elle correspond à la séparation entre l'organisation et son environnement, et entre le créateur et l'organisation créée : «La limite distingue également l'individu - comme organisation de l'individu - comme travailleur, [...] travail effectué comme membre d'une organisation (c'est-à-dire, à l'intérieur des conditions de la limite) à partir du travail effectué en tant qu'individu (c'est-à-dire du travail effectué en dehors des conditions de la limite). » Sur le plan pratique, elle correspond à ces actes par lesquels le créateur manifeste l'existence de son organisation comme, par exemple, l'inscription au registre du commerce ou la prise d'un abonnement téléphonique.

L'échange constitue le dernier élément nécessaire pour avoir une organisation complète. Il peut être de deux ordres : interne, c'est-à-dire entre composantes, ou externe avec l'environnement. S'il est nécessaire, il n'est pas toujours efficient dès le début, et Katz et Gartner indiquent qu'une organisation peut vendre à perte, pendant un certain temps, pour gagner des parts de marché. S'intéresser à cette période au cours de laquelle ces quatre composantes se mettent en place doit permettre aux chercheurs de mieux comprendre ce concept d'émergence et comment naissent les organisations. 
Enfin, le succès remporté actuellement par l'analyse en termes de processus, qu'il s'agisse du champ de l'entrepreneuriat ou de celui plus large des sciences de gestion, ne doit cependant pas faire oublier que si, pour l'entrepreneur ou le dirigeant, le chemin suivi est important, cela ne le dispense pas pour autant d'obtenir un résultat. Pour emprunter le langage des juristes, si le médecin ou l'avocat sont tenus à une obligation de moyens, l'entrepreneur ou le dirigeant, eux, sont tenus à une obligation de résultats à laquelle, malheureusement, ils ne se conforment pas toujours.

\section{Conclusion}

Ainsi, ces dernières années la recherche en entrepreneuriat est passée de travaux intégralement consacrés aux caractéristiques psychologiques du créateur à des réflexions sur la notion d'organisation émergente où son rôle devient mineur. Gartner (1993) recommande, pour progresser, de s'intéresser à autre chose qu'aux créateurs : "Je crois que "les créateurs" d'organisation sont une partie importante de l'émergence d'organisation mais je pense que nous pourrions voir quelques facteurs intéressants et importants, critiques vis-à-vis de ce phénomène si nous détournions une partie de notre attention de ces individus. » (p. 234). Pourtant, il ne faudrait pas aller trop loin dans cette voie et en arriver à parler de création en oubliant le créateur. Pasteur a mis fin à la croyance en la génération spontanée pour les organismes vivants ; il ne faudrait pas maintenant se mettre à croire à la génération spontanée des organisations. Elles émergeraient ainsi du néant par une sorte de concours de circonstances où la volonté humaine serait quasi absente ou, en tous cas, très minimisée. Ce qui donne naissance à une organisation, c'est avant tout la volonté humaine émanant d'un individu ou d'un groupe d'individus. Parleraiton aujourd'hui de Microsoft sans Gates ? Même l'intrapreneuriat n'existe pas sans l'existence de l'intrapreneur. Ce n'est pas l'organisation mère qui, spontanément, donne naissance à des bourgeons, c'est la volonté d'un intrapreneur. $3 \mathrm{M}$ n'aurait pas connu le succès avec les célèbres « Post It » s'il n'y avait eu la ténacité, l'opiniâtreté d'A. Fry. Kimberly (1979) qui, étudiant l'ouverture d'une nouvelle école de médecine, insiste sur le rôle déterminant joué par le créateur, en l'occurrence le premier doyen de cette école. Et le même Gartner (qui recommande d'oublier un peu le créateur), lorsqu'il propose un modèle d'organisation émergente nécessitant quatre propriétés, cite en premier l'intention, la volonté. Or, qui peut manifester une intention, faire preuve de volonté, si ce n'est le créateur? Son modèle montre bien l'impossibilité totale de se passer du créateur, de créer sans créateur.

On ne peut donc que suivre les sages prescriptions de Herron et Robinson (1993) lorsqu'ils recommandent, en présentant leur modèle, de ne 
pas oublier l'entrepreneur : « On espère que l'explication de ce modèle encouragera la recherche en entrepreneuriat à venir, qui cherche à examiner la cause de la performance de la nouvelle entreprise, à réintroduire "l'entrepreneur" comme centre d'intérêt ou un des centres d'intérêt de la recherche. » De même pour Shaver et Scott (1991): «Les circonstances économiques sont importantes ; les réseaux sociaux sont importants ; les équipes entrepreneuriales sont importantes ; la mercatique est importante ; la finance est importante ; même l'assistance de l'agence gouvernementale est importante. Mais aucun de ces éléments ne créera à lui seul une nouvelle entreprise. Pour cela, il faut une personne, dans l'esprit de laquelle toutes les possibilités se réuniront, qui croit que l'innovation est possible, et qui est suffisamment motivée pour mener à bien le travail jusqu'à son terme. »

L'étude du processus entrepreneurial ne doit pas faire oublier totalement celle de l'acteur, celle du créateur. Ce double centre d'intérêt (processus et créateur) constitue peut-être une voie vers une théorie de l'entrepreneuriat moins sujette à controverses que les diverses tentatives ayant déjà eu lieu. Elle reste cependant bien difficile à établir et pour Hofer et Bygrave (1992) neuf facteurs propres au processus entrepreneurial en font ressortir toute la complexité : «Le processus entrepreneurial : est initié par un acte humain volontaire, survient au niveau de la firme individuelle, implique un changement d'état, implique une discontinuité, est un processus holistique, est un processus dynamique, est unique, implique de nombreuses variables préalables, génère des conséquences qui sont extrêmement sensibles aux conditions initiales de ces variables. » Ces caractéristiques constituent un véritable défi pour les chercheurs.

\section{Bibliographie}

BULL, I. et G.E. WILLARD (1993), « Towards a theory of entrepreneurship », Journal of Business Venturing, vol. 8, $\mathrm{n}^{\circ} 3$, p. 183-195.

BYGRAVE, W.D. et C.W. HofER (1991), «Theorizing about entrepreneurship », Entrepreneurship Theory and Practice, hiver, vol. 16, $\mathrm{n}^{\circ}$ 2, p. 13-22.

COVIN, J.G. et D.P. SLEVIN (1991), «A conceptual model of entrepreneurship as firm behavior », Entrepreneurship Theory and Practice, vol. 16, $\mathrm{n}^{\circ} 1$, p. 7-25.

GARTNER, W.B. (1989), "Who is an entrepreneur? is the wrong question », Entrepreneurship Theory and Practice, vol. 13, $\mathrm{n}^{\circ} 4$, p. 47-67.

GARTNER, W.B. (1993), « Words lead to deeds : towards an organizational emergence vocabulary », Journal of Business Venturing, vol. 8, $n^{\circ} 3$, p. 231-239. 
GARTNER, W.B., B.J. BIRD et J.A. STARR (1992), «Action as if : differentiating entrepreneurial from organizational behavior », Entrepreneurship Theory and Practice, vol. 16, $\mathrm{n}^{\circ}$ 3, p. 13-31.

Greenberger, D.B. et D.L. SEXTON (1988), « An interactive model of new venture initiation », Journal of Small Business Management, vol. 26, $\mathrm{n}^{\circ} 3$, p. 1-7.

Guth, W.D., A. Kumaraswamy et M. McErlean (1991), « Cognition, enactment and learning in the entrepreneurial process », Frontiers of Entrepreneurship Research, p. $242-253$.

HERron, L. et R.B. JR. RoBinson (1993), «A structural model of the effects of entrepreneurial characteristics on venture performance », Journal of Business Venturing, vol. 8, $\mathrm{n}^{\circ} 3$, p. 281-294.

Hofer, C.W. et W.D. BygRAVE (1992), "Researching entrepreneurship », Entrepreneurship Theory and Practice, vol. 16, p. 91-100.

Hornsby, J.S., D.W. NaffZiger, D.F Kuratko et R.V. Montagno (1993), « An interactive model of the corporate entrepreneurship process », Entrepreneurship Theory and Practice, vol. 17, $\mathrm{n}^{\circ} 2$, p. 29-37.

KATZ, J. et W.B. GARTNer (1988), «Properties of emerging organizations », Academy of Management Review, vol. 13, $\mathrm{n}^{\circ} 3$, p. 429-441.

KIMBERLY, J.R. (1979), «Issues in the creation of organizations : initiation, innovation, and institutionalization », Academy of Management Journal, vol. 22, $\mathrm{n}^{\circ} 3$, p. 437-457.

LEARNED, K.E. (1992), «What happened before the organization? A model of organization formation », Entrepreneurship Theory and Practice, vol. 17, $\mathrm{n}^{\circ} 1$, p. 39-48.

MÉRIgOT, J.G. (1992), «Qu'est-ce que l'entreprise ?», Encyclopédie du Management, Paris, Vuibert.

SHAVER, K.G. et L.R. SCOTT (1991), « Person, process, choice : the psychology of new venture creation », Entrepreneurship Theory and Practice, ${ }^{\circ} 16$, p. 23-45.

ThÉVEnet, M. et J.L. VACHeTte (1992), Culture et comportements, Paris, Vuibert, Collection « Ressources Humaines ». 